\title{
Reading and Writing Skills of Scientific Articles for Undergraduate Students: Benefits and Challenges
}

\author{
Dyah Sunggingwati \\ Faculty of Teacher Training and Education \\ UniversitasMulawarman \\ Indonesia \\ sunggingwati@gmail.com
}

\begin{abstract}
This study aims to explore challenges and benefits attained by students when they read scientific articles for their purpose of research outline. Five English education students of the $5^{\text {th }}$ semester in Mulawarman University, Indonesia, participated in this study. They were required to search 12 scientific articles that had similar topic they were interested in. They were taught and demonstrated how to find out scientific articles, take a note, paraphrase and summarize. The findings obtained from the students' reflections and documents indicated that determining the topic and new vocabularies, highlighting important words, paraphrasing, and managing the time were complicated. However, as they became accustomed with the assignment, they knew more about scientific articles, gained new vocabularies, and were better in managing their time. They were content since the task had provided them insight for their mini thesis.
\end{abstract}

\section{Keywords-reading, scientific article, english student}

\section{INTRODUCTION}

Internet in the $21^{\text {st }}$ century is undeniable that allows people to gain new information, knowledge, and new technology. In education, the benefits of the Internet are various. Belisle, [1] claimed that learning is more active since the Internet allows students to have more experience with the websites. They can discover, explore and find the excitement. Therefore, the passive learning, which might be just in the form of listening to the teachers then shifts to more adventureful learning as they need to browse the Internet. The active learning will lead to independence in learning. Not only for teachers' teaching materials and for methodology, the use of internet also supports the development of independent learners [2]-[4].

Meanwhile, Internet has played an increasingly important role for foreign language/second language readers since online reading serves as the source of input for them [5]. Leu, et al. [6] has underlined that Internet offers minimum time for information and communication compared to the other media such as books or computers. According to Coiro, [7] electronic texts introduce new supports as well as new challenges that can have a great impact on an individual's ability to comprehend what people read.

Furthermore, for pre-service teachers in this study context, reading particularly scientific journal articles is uncommon. Since this is the first time for them to get to know the scientific articles, therefore, they struggle to comprehend them due to unfamiliar vocabularies and genre.

Reading comprehension literature has indicated that providing reading strategies such as reviewing, scanning, skimming, predicting, questioning, visualizing, and summarizing are reading comprehension strategies which can be taught to students. Previewing strategies can be used to activate background knowledge. Predicting strategies are employed to anticipate a story event or what might happen in a passage. Questioning strategies are applied to guide reading and to monitor understanding. Using visualizing strategies can develop students' representation of their passage understanding and summarizing strategies can be used to synthesize key ideas of the passage content [8], [9]. Demonstration and opportunities for students to use the strategies with teacher supervision are essential [10], [11].

Grabe, [12] reported that lack of academic vocabulary knowledge is a major factor that impaired students for better reading abilities or writing task. Further, they underlined that it is significant for teachers to consider how to implement effective writing process from text sources, and how to do effective paraphrasing.

Research on reading related to the Internet usually linked with writing skills. Villaume, [13] Investigated the influence of Cooperative Integrated Reading and Composition (CIRC) on students' writing in the Turkish context. A study group consisting of 16 students learning Turkish language in Estonia participated in this study. The author implemented the CIRC and analyzed it quantitatively and qualitatively. The students' behavior regarding reading activities showed that there were highest frequency of vocabulary and simple skills such as scanning and skimming, using background knowledge text, finding meanings and places of certain information in the text. Meanwhile, there are more mistakes on vocabulary and concepts, phonemes and syllables level, sentence level, spelling and punctuation, and mistakes related to transfer from the mother tongue.

Integration of reading and writing was also researched. A needs survey was also conducted by Cho and Brutt-Griffler, [14] at middle school level in Korea. About 93 students and 69 students were involved in a three-week intervention with the objective of summary writing improvement. The results revealed that the students perceived that the guidance for 
integrated reading and writing skill is necessary. The students lacked vocabulary so that they were more challenged in paraphrasing before summary writing. The authors also indicated that the intervention improved summary writing skills but not reading ability due to insufficient time of a threeweek intervention.

In a recent study [15], reviewed studies on students' writing found out that in the academic context, the students were encountered with challenges to write from sources but create particular strategies to deal with. Knowledge and experience, types of texts and tasks impact on students' writing performance. The authors also highlighted that instruction could assist those difficulties. One of the important issues emerged from their review was that the students at the secondary level could be guided to do and write the independent research from library and Internet resources.

Previous research indicated that students could be taught to summarize from reading the sources from the Internet. Therefore, this study will explore the challenges and benefits obtained by students when they read scientific articles for their purpose of research outline.

\section{METHOD}

\section{A. The participants}

Five English Education students of the 5th semester in Mulawarman University, Indonesia, participated in this study. They were selected on the basis of their competence to generate research outline after the advanced reading and writing courses were completed. In this course, they were required to search 12 scientific articles that had similar topic. They were trained how to find out scientific articles, take a note, paraphrase and summarize. Therefore, at the end of the course, they had 12 articles that had been highlighted, paraphrased, and summarized; and their reflections. Furthermore, for pre-service teachers in this study context, reading particularly the scientific journal articles is uncommon and thus this was the first time for them to get to know the scientific articles.

\section{B. The training procedure}

The students were taught how to browse and search scientific article from reliable sources. The students were explained about the journal articles and the benefits of using them for research ideas. Next, I showed the students the websites that they could browse and download reliable free access journals on. The explanation of how to search the articles they were interested in through the article abstracts and suggestion to read the abstract before they downloaded to ensure the article was the one they wanted to be provided. I also pointed out that abstract could provide general information of the article content. Offering open access links and demonstration of how to search article using keywords was also given.

In skimming phase, the students were asked to read the articles quickly to get the sense of the article content. Next, they needed to tell their peer what the article was about. Completing this, the students were asked to do the scanning.
While doing scanning, the students had to highlight the keywords that they might think important. I also informed them that from the highlighted keywords, they needed to be able to link the keywords into their own sentences which I called as note-taking. This case had to be done for each paragraph of the whole article.

The following phase was paraphrasing. The students were required to take the notes they made and asked to paraphrase each paragraph. How and what to be paraphrased were demonstrated. After the paraphrasing was carried out, the last phase was summarizing. The students had to blend their paraphrasing into a summary of the article. Therefore, the students would have twelve articles with paraphrasing and summary for each of them.

\section{Data and data analysis}

The data were gathered from students' reflections and documents of five students. The reflective journal that could employ Bahasa Indonesia or English or mixed, was less formal as a way to express what they thought about the article, and was accomplished after each article was fulfilled. The journal reflected the difficulties they faced and advantages they gained during the completion for each article including any strategies they dealt with their inconvenience. The documents are the outline of the research proposals the students made after completing the assignments. All the responses were analyzed based on the themes emerged from the responses. The chucks of similar responses in each phase were merged to achieve one similar theme.

\section{FINDINGS AND DISCUSSION}

\section{A. Findings from reflective journals}

1) Article-1 and Article-2

The findings from the reflective journals indicated that the first article was the most challenging for the students. Determining the topic of the articles was uneasy for the students, particularly when they encountered unfamiliar words. This case occurred not because of the content of the articles but their first experience in dealing with scientific articles.

This task is really under pressure assignment...I got culture shocked. I never complete assignment until stayup-late and this is the first article and I don't know the rest. Since the following day is my presentation in another course and it is my second priority. I usually prioritize my following assignment. This case means that this article task is my big deal. Searching for articles that followed the requirement such as being "update, not less than 10 pages, and not conference proceeding, was hard. However, there is a positive point from this task that I could type with 10 fingers and got new vocabulary through this "Holy assignment" (S-1)

The student indicated that the assignment was complicated as she experienced culture shock, needed to stay-up-late, and encountered confusion. However, she also achieved some new vocabularies. In a more detail information of how the students dealt with the assignment, one student reported as follow: 
When I started working on my assignment, I faced unfamiliar vocabulary so it took time to finish the noting. I have more new vocabularies. The way the author delivered the article was clear and could be understood. I felt that my fingers felt apart after completing the noting as the writing font was very small. Next, paraphrasing was confusing for me... I did not know what I had to do with the paraphrasing as I was afraid of plagiarism. So when doing paraphrasing, the dictionary was ready for searching synonym. It took a long time for paraphrasing. Then, summarizing was easy as I understood the content. As I finished all, I felt released as if I drank sprite. (S-2)

The reflection implied that new vocabulary was uneasy for the students, so they needed to be ready with the dictionary. Paraphrasing was challenging as the student was unsure whether it was correct paraphrasing and it took more time to do it. The summary was not as complicated as the paraphrasing. The students also reflected how they finally found out the topic they wanted and their ability in managing their time. One of them reported as follow:

Determining the topic was hard for me as I had to change three times and finally, I found out the topic of CLIL approach but then the recent one was not available. I found the recent article but the content was not the one I wanted. Limited internet access also created more frustration for me. However, I got a new knowledge such as where I could find the international articles, and the one with open access or not. I think it was worth. From this (tiring) fun assignment I felt that I had to improve myself, I am already in the fifth semester and not the time to compete for the assignment in a rush. Now, I realize that I had to be better to manage my time. (S-5)

The reflection revealed that searching article required several attempts. Matching the recent one with the students' interest led to frustration but the students also got benefits during the process, including self-improvement in time management. In the second article, four students indicated that they got used to know the content of their articles but had problem in highlighting as one reflected:

I could work more smoothly for the second article than the first one but still could not manage my time properly. The article was relatively easy to be understood but I had difficulty in highlighting as I thought that every word was important. (S-5)

The students still encountered unfamiliar words but they felt much better in doing the second assignment than the first article. Highlighting was the major problem as the vocabulary was limited.

\section{2) Article-3 to Article-6}

The reflections of the article- 3 to article- 6 were similar among the students that they got used to with the assignments.

By the fourth article, I just realized the strengths of the assignment; one of them was the effect of paraphrasing that allowed to know more synonyms. I unconsciously remember some synonyms such as situation circumstances, competence - proficiency - skill-ability - capability and etc. (S-1)

This phenomenon indicated that paraphrasing could improve vocabularies. In addition, the students could complete the assignment soon.

I was excited to complete this assignment because my friends and I planned to have a picnic in Balikpapan. I could complete the article sooner and perhaps this is the fastest one than the previous ones. I did not have challenges with the fifth article. (S-3).

The sixth article did help me a lot in highlighting, and noting because the language was easy to be understood, the font was big enough even though it had many pages, I was still excited to do it. I did not find many difficulties in article six (S-4)

These two reflections indicated that the students had a fewer difficulties in completing the assignments. Their time to do this task was shorter, therefore, there was an improvement in managing their time to finish the task.

\section{3) Article-7 to Article-9}

Almost all students indicated that they started getting bored with the assignment.

I could finish article- 8 and 9 in a week. So, it means two articles in one week. This $9^{\text {th }}$ article was not thicker than article-8, but when I did it, I felt very bored and I was getting sick of it even it was tiring. I wanted to quit but it was already article-9. I needed three more articles. (S$1)$.

The students could work with fewer complexities as they could finish the task of two articles within a week. However, feeling bored emerged. Meanwhile, another student indicated that she was happy with her improvement in doing the assignment.

This is my eighth article and I felt that I made a good progress in noting, paraphrasing, summarizing which are better than the previous articles. So, I don't need to make many reflections as I felt the improvement to do it. (S-2)

Although the students were bored with the task, at the same time they could realize the improvement of doing the task.

\section{4) Article-10 and Article-11}

All students indicated that they did not have problems in completing the assignment. However, since the students had to finish the other tasks from the other courses, most of them tended to do this task at the last minute.

For this time, although I had a bit problem to find article-11, everything ran smoothly, no difficulties in noting and highlighting. However, I had the other assignments from the other courses that made me learn and I was a bit lazy to do paraphrasing. (S-3).

I am confused what I had to write. I got used to with the article even it was tiring, boring, sick of it and mentally exhausted, but I accepted as I had to do it. (S-1) 
These two reflections revealed that the students understood that they had fewer and fewer difficulties but the fatigued increased.

\section{5) Article-12}

Some students reported their experiences from the first article to the last one.

In this article-12, I highlighted, paraphrased, and summarized for the last time. Thank God! Thank to the lecturer for the experiences and valuable guidance in this semester. It was a very impressive assignment. I hope that this experience and effort I had done could yield a good result for my mini-thesis. Thank you (S-5)

Overall, I got used to read, highlight, paraphrase, and summarize the article. If the other people bring a novel in their leisure time, I bring article whenever I go. If I have a few minutes, I made use of it by doing the task bit by bit. I also know how to deal with my mood by playing a game. It was very effective when my mood was dropped during the task (S-1)

The reflections implied that the students worked hard to do the assignment but the experiences indicated that they could take positive points during the process of completion.

\section{B. Findings from the documents}

The document findings were gathered from the outline of the research topic. Generally, all of the students were able to generate research outline based on their article assignments. All of them provided the introduction/ background of the study, the purpose, the research questions, and some information of the design and participants. Among these, one noticeable feature is introduction section as this part requires students to argue why their investigation is necessary to be conducted. In this case, they need to consider both the literature and previous research. Therefore, the findings from the document focus on the research outline of the introduction part.

Introduction: all of the students wrote down the introduction or background of their outline with the claims of the experts. The students could combine the claims from different experts but they needed more empirical studies related to the topic. In addition, the students could link the literature and the context where they could conduct the investigation. One example is given in the following paragraph.

Content-language integrated learning is an educational approach that supposed to help the students to improve their knowledge of the content which is being taught by their teacher using the foreign language and subconsciously advance their students' foreign language. Morton, [16], as cited in van Kampen et al. [17], claimed that CLIL is not connected but it integrates the subject and language teaching pedagogies. In Indonesia, there are so many international-based schools. It means that in those school, there must be multilingual teaching and learning activity. In Samarinda, there were many International-based schools. Unfortunately, the bilingual program in public schools in Samarinda has been erased a few years ago for some reasons such as pretty high cost, unprepared teachers, facilitates and so on. Therefore, there are only several private schools which still adopt the bilingual program and these schools are rarely found. (S-5)

The students could collaborate the definition contentlanguage integrated learning (CLIL) with one of the schools that uses English as a medium of instruction. They also indicated the possibility of its implementation. Among those five students, one of them could identify the link between the experts and previous studies as follows:

Moreover, extensive reading can give chances for fun reading and learning since it is concentrated on holistic language proficiency and can encourage language acquisition. It also has a great position for vocabulary acquisition which means its effectiveness can lead the readers to acquire new vocabulary from context [11], [18], [19]. In another side, its correlation with the topicfamiliarity-based is that the content can be influenced by topic-familiarity and interest because the readers usually gather the content knowledge based on their point of view and attraction [20]. So, I guess this aspect is a great match for extensive reading. Horiba, [20] also added in their study that topic-familiarity has a big role in incidental vocabulary acquisition (S-1).

The above paragraph reveals that the student could identify what needs to be done from the literature and previous research with the recent references. She wrote what claims that were available from the literature followed by evidence from the research.

\section{Discussion}

The key findings of this study have revealed that the students encountered some challenges and gained some benefits from the assignment. Since they needed to be involved in different phases such as seeking scientific articles, skimming and scanning, highlighting, noting, paraphrasing, and summarizing, their challenges and advantages varied. In searching the topic of their articles, the students had to think and sometimes changed their topic because it did not fulfill the requirement such as the recent articles, the number of pages that should be more than 10 excluded the references and appendix, it is not conference proceeding and the 12 articles should be relevant each other. The students experienced this thing, particularly when they had to do it for the first and second articles. All students had to spend more time to deal with the assignment.

In addition to this case, the new vocabularies were unfamiliar for them contributing to their complexities to accomplish the tasks. Grabe, [12] has identified that the lack of academic vocabulary was the major problem for ESL students, particularly in reading and writing skill. Preparing dictionary and looking for the synonyms assisted them to cope with such problems. Because of the lack of vocabulary, it could be hypothesized that the students confronted more difficulties in highlighting. Although it did not occur for all students, highlighting was uneasy as the students thought all words were necessary to be put in the note-taking. Paraphrasing was 
perhaps the most challenging to do than the other phases. This case supports previous research by Cho and Brutt-Griffler [14] mentioning that the lack of vocabulary led to the difficulties in paraphrasing. Furthermore, they had a problem of time management that was they had to finish their duty in time.

The students were getting used to with their tasks as they accomplished the article-3 to article-6. They became accustomed to the paraphrasing that enhanced their vocabularies as they became familiar with and they could finish their project earlier. In the article-7 to article-9, the students reached the feeling of bored and had fewer complexities to do their work. The other assignments from the other courses offered more students' depressed. This case could be seen from their reflection of doing article-10 and 11, but they felt released when they had worked on article-12 as the last one.

The findings from the research outline showed that the students could combine the claims from different experts, link the literature and the context, and indicate the possibility of its implementation. Furthermore, the students could identify the link between the experts and previous studies with recent references. They wrote the claims which were available from the literature followed by the evidence from the empirical evidence. This finding was linked with a review study by Cumming, et al. [15] who identified that the students can be taught to write independently from the Internet sources.

\section{CONCLUSION}

This study implied that the students could be guided by the task that they thought was dreadful. Their early efforts to do the assignment were tough and time-consuming. However, as they were accustomed to the tasks then they encountered fewer difficulties at the end. They could manage their time to work on the task and achieve some ideas for their mini-thesis. They were eventually delighted with what they have done and achieved in the course. Although the task was demanding and complex, the students gained the benefits not only in their learning and research outline but also their life in terms of time- and self- management.

\section{ACKNOWLEDGEMENT}

I would like to thank Islamic Development Bank Project of Mulawarman University for the support to provide the grant to publish this paper.

\section{REFERENCES}

[1] R. Belisle, "E-mail activities in the ESL writing class," Internet TESL J., vol. 2, no. 12, pp. 56-79, 1996.

[2] K. Schwienhorst, Learner Autonomy and CALL Environments. London: Routledge, 2008.

[3] A. Vanijdee, "Thai distance English learners and learner autonomy," Open Learn., vol. 18, no. 1, pp. 75-84, 2003.
[4] B. Varışoğlu, "Influence of cooperative integrated reading and composition technique on foreign students' reading and writing skills in Turkish," Educ. Res. Rev., vol. 11, no. 12, pp. 1168-1179, 2016.

[5] N. J. Anderson, "Scrolling, clicking, and reading English: Online reading strategies in a second/foreign language," The Reading Matrix, 2003. [Online]. Available: http://www.readingmatrix.com/articles/anderson/article.pdf. [Accessed: 09-Apr-2013].

[6] D. J. Leu, E. Forzani, C. Burlingame, N. Kulikowich, Jonna and Sedransk, J. Coiro, and C. Kennedy, "The new literacies of online research and comprehension: Assessing and preparing students for the 21st century with Common Core State Standards," Qual. Read. Instr. age Common Core Stand., pp. 219-236, 2013.

[7] J. Coiro, "Exploring Literacy on the Internet: Reading comprehension on the Internet: Expanding our understanding of reading comprehension to encompass new literacies," Read. Teach., vol. 56, no. 5, pp. 458-464, 2003.

[8] L. Burke, J. Fiene, M. J. Young, and D. K. Meyer, "Understanding the why behind the how in reading instruction," Educ. Forum, vol. 72, no. 2, pp. 168-176, 2008.

[9] B. M. . Taylor, P. D. Earson, G. E. Garcia, K. Stahl, and E. B. Bauer, "Improving students' reading comprehension," in Reading research at work: Foundations of effective practice, K. A. D. Stahl and M. C. McKenna, Eds. New York, NY: Guilford Press, 2006, pp. 303-315.

[10] N. K. Duke and P. D. Pearson, "Effective Practices for Developing Reading Comprehension," J. Educ., vol. 189, no. 1/2, pp. 107-122, 2008.

[11] D. Gablasova, "Learning and retaining specialized vocabulary from textbook reading: Comparison of learning outcomes through L1 and L2," Mod. Lang. J., vol. 98, no. 4, pp. 976-991, 2014.

[12] W. Grabe and C. Zhang, "Reading and writing together: A critical component of English for academic purposes teaching and learning," TESOL J., vol. 4, no. 1, pp. 9-24, 2013.

[13] S. K. Villaume and E. G. Brabham, "Comprehension instruction: Beyond strategies.(questions and answers)," Read. Teach., vol. 55, no. 7, pp. 672-676, 2002.

[14] H. Cho and J. Brutt-Griffler, "Integrated reading and writing: A case of Korean English language learners," Read. a Foreign Lang., vol. 27, no. 2, pp. 242-261, 2015.

[15] A. Cumming, C. Lai, and H. Cho, "Students' writing from sources for academic purposes: A synthesis of recent research," J. English Acad. Purp., vol. 23, pp. 47-58, 2016.

[16] T. Morton, "Using a genre-based approach to integrating content and language in CLIL," in Language use and language learning in CLIL classrooms, C. Dalton-Puffer, T. Nikula, and U. Smit, Eds. Amsterdam: John Benjamin, 2010, pp. 81-104.

[17] E. van Kampen, W. Admiraal, and A. Berry, "Content and language integrated learning in the Netherlands: teachers' self-reported pedagogical practices," Int. J. Biling. Educ. Biling., pp. 1-15, 2016.

[18] A. Pellicer-Sánchez, "Incidental L2 vocabulary acquisition from and while reading: An eye-tracking study," Stud. Second Lang. Acquis., vol. 38, no. 1, pp. 97-130, 2016.

[19] F. Teng, "Incidental vocabulary acquisition from reading-only and reading-while-listening: a multi-dimensional approach," Innov. Lang. Learn. Teach., pp. 1-15, 2016.

[20] Y. Horiba and K. Fukaya, "Reading and learning from L2 text: Effects of reading goal, topic familiarity, and language proficiency," Read. a Foreign Lang., vol. 27, no. 1, pp. 22-46, 2015. 